\title{
EFEITO DO PESO AO ABATE DE CORDEIROS SANTA INÊS E BERGAMÁCIA SOBRE AS CARACTERÍSTICAS FÍSICO-QUÍMICAS DA CARNE ${ }^{1}$
}

\author{
Maria Cristina BRESSAN ${ }^{2, *}$, Osni Vieira $\mathrm{PRADO}^{2}$, Juan Ramon Olalgaquiaga PÉREZ², \\ Ana Lúcia da Silva Correa LEMOS², Sarita BONAGURIO²
}

\begin{abstract}
RESUMO
Foram utilizados 36 cordeiros machos inteiros, sendo 24 da raça Santa Inês e 12 da raça Bergamácia, os quais entraram no experimento com $15 \mathrm{~kg}$ e foram distribuídos aleatoriamente nos grupos de peso ao abate (GPA) de 15, 25, 35 e $45 \mathrm{~kg}$. O objetivo do trabalho foi avaliar o declínio do $\mathrm{pH}$ post-mortem, cor (CIE L*a*b*), perda de peso por cozimento (PPC) e força de cisalhamento (FC) nos músculos longissimus dorsi (LD) e semimembranosus (SM). Os parâmetros estudados foram submetidos à análise de regressão. $\mathrm{O}$ valor de $\mathrm{pH}$ para ambos os músculos foi expresso através de equações de regressão do tipo exponencial, apresentando uma rápida queda nas primeiras horas, seguido de estabilização. No músculo LD foi encontrado que a glicólise desenvolveu-se mais rapidamente com o aumento do peso. A análise dos componentes da cor para ambos os músculos estudados apresentaram o mesmo comportamento. A raça Bergamácia apresentou maior índice de luminosidade $\left(L^{*}\right)$, ou seja, maior brilho. O valor de $L^{*}$ diminuiu, o teor de vermelho $\left(a^{*}\right)$ aumentou e o teor de amarelo $\left(b^{*}\right)$ diminuiu linearmente em relação ao aumento do peso. A análise geral dos componentes da cor indicou que a carne de cordeiro é mais escura com o aumento do peso. Os resultados das análise de PPC e FC foram similares entre as raças e GPA, os valores obtidos para PPC foram de $28,0 \pm 2,5 \%$ (LD) e $30,5 \pm 4,1 \%$ (SM), para FC foram de $2,6 \pm 0,6 \mathrm{~kg}$ (LD) e $2,8 \pm 0,7 \mathrm{~kg}(\mathrm{SM})$.
\end{abstract}

Palavras-chave: cordeiro; carne; qualidade.

\section{SUMMARY}

EFFECT OF THE SLAUGHTER WEIGHT ON THE PHYSICALCHEMICAL CHARACTERISTIC OF BERGAMÁCIA AND SANTA INÊS LAMBS MEAT. Thirty-six whole male lambs were used, being twenty-four of Santa Inês breed and twelve of Bergamácia breed, which weighed $15 \mathrm{~kg}$ at the very beginning of the experiment, and were randomized allocated into slaughtering weight groups (SWG) of $15,25,35$ and $45 \mathrm{~kg}$. The objective of this work was to evaluate the $\mathrm{pH}$ post-mortem decline, color $\left(\mathrm{CIEL}^{*} \mathrm{a}^{*} \mathrm{~b}^{*}\right)$, cooking weight loss (CWL) and shearing force (SF) in the longissimus dorsi (LD) and semimembranosus (SM) muscles. The examined parameters were submitted to a regression analysis. The value of $\mathrm{pH}$ for both muscles was expressed by regression equation of exponential type, presenting a fast fall in the first hours, followed by stabilization. The LD muscle glycolisis rate was found to develop more quickly with an increase in weight. The analysis for color components for both examined muscles presented the same behavior. The breed Bergamacia presented larger index lightness $\left(L^{*}\right)$, that is to say, larger brilliance. The value of $L^{*}$ decreased, the red content $\left(a^{*}\right)$ increased and the yellow content $\left(b^{*}\right)$ linearly decreased as the weight increased. The general analysis for color components had indicated that lamb becomes darker with weight increase. The results of CWL and SF analyses were similar in

${ }_{1}^{1}$ Recebido para publicação em 10/05/00. Aceito para publicação em 10/09/01.

${ }_{2}^{2}$ Universidade Federal de Lavras. Departamento de Ciência dos Alimentos. Cx. Postal 37, CEP 37200-000, Lavras - MG.

${ }^{*}$ A quem a correspondência deve ser enviada. both breeds and SWG, the values for CWL were $28.0 \pm 2.5 \%$ (LD) and $30.5 \pm 4.1 \%(\mathrm{SM})$ and for SF $2.6 \pm 0.6 \mathrm{~kg}(\mathrm{LD})$ and $2.8 \pm 0.7 \mathrm{~kg}$.

Keywords: lamb; meat; quality.

\section{1 - INTRODUÇÃO}

No Brasil, a maioria dos ovinos destinados ao abate são comercializados com peso elevado, pois o produtor é remunerado em função do peso ao abate. Assim, o produtor vende ovinos mais pesados para obter maior lucratividade. Esses animais mais pesados, geralmente são mais velhos e possuem maior percentual de gordura na carcaça.

As características de qualidade mais importantes na carne vermelha são aparência (cor, brilho e apresentação do corte) responsável pela aceitação do consumidor no momento da compra e maciez que determina a aceitação global do corte e do tipo da carne, no momento do consumo. Esses atributos ou características físicas apresentam variações que estão associadas a vários fatores, tais como: diferenças na idade e/ou peso ao abate, manejo pré e pós-abate e tipos de raças.

Atualmente, o mercado consumidor apresenta elevada exigência quanto à qualidade das características físicas da carne, o que torna necessário o conhecimento dessas características nas diferentes faixas de peso dos ovinos destinados ao abate.

As raças ovinas Santa Inês e Bergamácia, que vêm apresentando potencial para cortes, são de grande porte, adaptam-se com facilidade aos climas mais quentes e são bastante prolíferas. No entanto, os artigos encontrados na literatura a respeito da qualidade físico-química da carne de cordeiros Santa Inês e Bergamácia são escassos. Também raros, senão inexistentes, são os trabalhos que estudaram a influência do peso ao abate sobre os parâmetros de qualidade nessas raças.

A velocidade da queda do $\mathrm{pH}$ após a morte, causada pelo acúmulo de ácido láctico, resultado das reações químicas post mortem, constitui um dos fatores mais marcantes na transformação do músculo em carne, com decisiva importância na qualidade futura da carne e dos produtos preparados a partir dela [32].

$\mathrm{O} \mathrm{pH}$ final $\left(\mathrm{pH}_{\mathrm{f}}\right)$ do músculo, medido às 24 horas post mortem é outro fator que também exerce influência sobre vários aspectos na qualidade da carne, por exemplo, capacidade de retenção de água (CRA), perda de peso por cozimento (PPC) e força de cisalhamento [8]. Bem como as propriedades organolépticas maciez, suculência, flavour, aroma e cor [14]. 
Quando o $\mathrm{pH}$ atinge valor menor do que 6,0 durante a primeira hora decorrida do abate com a temperatura do músculo ainda alta, próxima aos $35^{\circ} \mathrm{C}$, tem-se indicação de carne potencialmente PSE (pálida, flácida e exsudativa), que proporciona coloração pálida com intensa exsudação. Essa anomalia é comum em carne suína [10,13], menos freqüente em peito de perus [7] e há indícios de sua ocorrência em frangos [11]. Todavia, se $\mathrm{o} \mathrm{pH}$ diminuir pouco após decorrido as primeiras horas do abate e permanecer com valor acima de 6,0 completadas as 24 horas post mortem, tem-se indicação de carne DFD (escura, firme e seca) caracterizada por elevada capacidade de retenção de água, coloração escura e vida-de-prateleira reduzida [3].

Considerando a influência do peso ao abate de espécies animais de carne vermelha sobre o $\mathrm{pH}$ final, os resultados são contraditórios. TUMA et al. [48], trabaIhando com novilhos abatidos com diferentes pesos, encontraram menor valor de $\mathrm{pH}_{\mathrm{f}}$ em animais com maior peso ao abate ou idade mais avançada. Em cordeiros, PINKAS et al. [35], estudando grupos de animais abatidos com 22 e 30 semanas de idade, não observaram diferenças significativas quanto ao $\mathrm{pH}_{\mathrm{f}}$. Contudo, DEVINE et al. [14], comparando cordeiros em crescimento com peso semelhante ao abate, mas com idade diferente, constataram que $\mathrm{o} \mathrm{pH}_{\mathrm{f}}$ dos cordeiros mais jovens foi ligeiramente menor. Por outro lado, SAÑUDO et al. [39], analisando três grupos de peso de carcaça de cordeiro $(8,1 ; 10,2 ; 13,4 \mathrm{~kg})$, verificaram que o grupo de carcaça mais pesado apresentou valor de $\mathrm{pH}_{\mathrm{f}}$ mais elevado, do que o grupo de carcaça de peso médio e leve, os quais foram similares.

Os valores de $\mathrm{pH}_{\mathrm{f}}$ em carnes podem sofrer alterações através do uso de drogas ou por condições de estresse pré-abate aos quais os animais são submetidos. Em ovelhas, o emprego de injeções pré-abate de epinefrina resultam músculos que apresentam valores de $\mathrm{pH}$ que variam de 5,6 a 7,0. As análises de qualidade da carne, nessa faixa de $\mathrm{pH}$, mostram que a perda de peso por cozimento diminuiu linearmente com o aumento do $\mathrm{pH}_{\mathrm{f}}$. $\mathrm{E}$, para a medida de maciez objetiva, é observada uma alta correlação linear quadrática negativa, onde a maior dureza ocorreu com valor em pH 6,0 [8].

Em ovinos, variações no $\mathrm{pH}$ em função da susceptibilidade ao estresse, que pode afetar a velocidade de queda do $\mathrm{pH}$ e $\circ \mathrm{pH}_{\mathrm{f}}$, foram descritas raramente. SINNETT-SMITHM et al. [45], estudando as raças East Friesland, Oxford e Texel, encontraram valores de $\mathrm{pH}_{\mathrm{f}}$ de 5,69, 5,64 e 5,63, respectivamente, sem diferença significativa. Outros autores como DRANSFIELD et al. [15], trabalhando com as raças Dorset Down e Suffolk, e OSÓRIO et al. [31], avaliando as raças Aragonesa, Ojinegra e Roya, não observaram diferenças estatisticamente significativas. No entanto, PEREZ et al. [34], acompanhando a queda do $\mathrm{pH}$ post mortem das raças Santa Inês e Bergamácia, verificaram diferenças significativas no $\mathrm{pH}$ às 6,12 e 18 horas. A raça Santa Inês apresentou valores de $\mathrm{pH}$ mais elevados em relação à Bergamácia. Entretanto, a diferença sig- nificativa de $\mathrm{pH}$ entre os músculos biceps femoris e longissimus dorsi foi averiguada apenas para a medida de $\mathrm{pH}$ às $6 \mathrm{~h}$, onde o músculo biceps femoris apresentou-se com maior valor.

A cor da carne é o índice de frescor e qualidade mais óbvio para o consumidor [40]. Normalmente carnes escuras são rejeitadas pelo comprador, que associa essas a carnes velhas ou carnes oriundas de animais mais maduros, portanto com carne dura. Entretanto, essa relação nem sempre é verdadeira, pois animais abatidos com pouca reserva de glicogênio não atingem valores de $\mathrm{pH}$ suficientemente baixos para produzir colorações normais, independente de sua idade e maciez [38].

Variações extremas na cor são relatadas principalmente em suínos onde cortes de carnes escuros possuem capacidade de retenção de água elevada (DFD) e carnes pálidas com capacidade de retenção de água baixa (PSE). Nesses dois tipos, o aspecto é indesejável ao consumidor e as propriedades sensoriais são desagradáveis [33]. Em ovinos, a anomalia PSE não tem sido relatada, no entanto existem incidências de carnes DFD [3].

A cor da carne depende do $\mathrm{pH}$ e da velocidade das reações químicas post mortem (glicólise). Quando o animal é submetido a estresse no pré-abate, ocorre uma redução da quantidade de glicogênio muscular. Isso resulta em um $\mathrm{pH}_{\mathrm{f}}$ elevado (acima de 6,0), o que torna mais ativas as citocromoxidases das mitocôndrias. Assim, um aumento no consumo de oxigênio pode aumentar a concentração de mioglobina desoxigenada, resultando em carnes de cor escura [3, 40].

As características da cor do músculo também podem ser afetadas pela espécie [6], sexo [43], estresse pré-abate [3, 4], idade do animal [39] e tratamento pósabate [17].

SAÑUDO et al. [39], comparando 3 grupos de pesos de carcaça com 8,1; 10,2 e 13,4kg, observaram que a estimativa para $L^{*}$ (índice de luminosidade) não mostrou diferença entre os grupos de peso de carcaça leve $(48,15)$ e médio $(47,20)$, as quais foram diferentes do grupo de carcaça pesado $(45,61)$, ou seja, mais escuro. Para a estimativa do valor $a^{*}$ (teor de vermelho), as carcaças mais leves $(13,94)$ apresentaram uma menor medida quando comparada com os valores de carcaça intermediária $(15,66)$ e pesadas $(16,95)$. E os resultados para estimativa de $b^{*}$ (teor de amarelo), mostraram que carcaças com peso intermediário $(6,86)$ possuem maior valor, do que carcaças leves $(5,90)$ e pesadas $(6,02)$.

Quanto à raça, PEREZ et al. [34] não relataram diferenças para os valores de medida de cor pelo sistema CIE, L* $(32,58-32,78) a^{*}(15,58-15,97) b^{*}(3,58-3,55)$, entre as raças Santa Inês e Bergamácia e músculos longissimus dorsi e bíceps femoris. Entretanto, OSÓRIO et al. [31] encontraram diferença para os valores de $L^{*} a^{*} b^{*}$ entre raças ovinas, tanto para o músculo longissimus dorsi, como para o triceps. 
A perda de peso no cozimento é uma medida importante de qualidade, pois está associada ao rendimento da carne no momento do consumo [32]. Essa é uma característica influenciada pela capacidade de retenção de água nas estruturas da carne [8]. A gordura existente na carne é derretida por ação do calor, que é registrada também como perda no cozimento [32].

Os cruzamentos Suffolk x Finnish-Southdown e Suffolk x Rambouillet [47], Targhee e Suffolk x Targhee [26] e as raças Santa Inês e Bergamácia [34], não apresentaram diferenças nos valores de PPC. Já, OCKERMAN et al. [29], trabalhando com cordeiros das raças Barbados, St. Croix (tipo pêlo) e nativos da Flórida e cruzados com Suffolk (tipo lã), encontraram diferença na PPC entre as raças deslanadas, mas não encontraram o mesmo nas raças lanadas e quanto ao tipo.

Os resultados para PPC em função do peso ao abate de ovinos são contraditórios. LLOYD, SLYTER, COSTELLO [26], trabalhando com cordeiros de dois grupos de peso, classificados como leve $(54 \mathrm{~kg})$ e pesado $(64 \mathrm{~kg})$, não encontraram diferenças para os resultados de PPC medida no músculo longissimus dorsi, obtendo valores de 15,49 e 16,75\%. KADIM et al. [20] encontraram tendência semelhante, avaliando linhagens ovinas selecionada para alta $(25,92 \%)$ e baixa $(25,99 \%)$ gordura na carcaça. Entretanto, KEMP et al. [23] avaliando 60 cordeiros (30 inteiros e 30 castrados) provenientes do cruzamento de carneiros Hampshire com oveIhas mestiças, sacrificados ao atingir peso ao abate prédestinados de 36,45 e $54 \mathrm{~kg}$, observaram que a PPC foi maior nos ovinos mais pesados. Esses resultados foram confirmados por KEMP et al. [21] em cordeiros originados do cruzamento de Hampshire vs (Suffolk $x$ Rambouillet). Da mesma forma, SCHÖNFELDT et al. [42] observaram que no músculo semimembranosus de ovinos e caprinos, a PPC aumenta com a idade. Pesquisando cordeiros da raça Katakatchanska com idade ao abate de 22 e 30 semanas, PINKAS et al. [35] encontram no músculo supraspinatus um aumento na PPC em ovinos com maior idade ao abate. Os valores encontrados foram 19,9 e $23,7 \%$, respectivamente. Esses autores acreditaram que a maior PPC nos cordeiros mais pesados ocorreu devido à maior quantidade de gordura presente nos tecidos.

Comparando tipos de alimentação, ASGHAR, YEATES [5] encontraram que cordeiros mantidos em diferentes planos nutricionais, submantença, matença e ad libitun apresentaram diferença para a PPC no músculo longissimus dorsi, onde uma menor PPC foi detectada nos animais em restrição alimentar $(29,83 \%)$ e maior nos cordeiros em ad libitun (35,87\%), os quais apresentaram maior quantidade de gordura. No entanto, NOTTER, KELLY, BERRY [28] não obtiveram diferença na PPC em cordeiros mantidos em sistema de manejo confinado $(31,5 \%)$, semiconfinado $(31,3 \%)$ ou a pasto $(31,6 \%)$.

BABIKER, EL KHIDER, SHAFIE [6] e SCHÖNFELDT et al. [42], comparando músculos longissimus dorsi de caprinos e ovinos, encontraram menor PPC em caprinos do que em ovinos. Os autores atribuíram esses resultados a maior quantidade de gordura presente no músculo dos ovinos.

A textura, para os vários tipos de carnes, é o critério de qualidade mais importante. Embora seja ampla a faixa de aceitação de maciez pelos consumidores, é certo que há vantagens para a carne mais macia quando os outros fatores são constantes. Os demais fatores que contribuem para as características organolépticas da carne incluem suculência e sabor [11].

As variações encontradas na maciez são decorrentes de vários fatores, tais como: raça dos animais [21, $23,27,30]$, peso ou idade ao abate [34, 39], tipos de músculos [1, 42], sistema de manejo [27, 28, 36] e condições de pré e pós-abate.

Considerando os trabalhos que estudaram o efeito da raça e peso ao abate sobre a força de cisalhamento (medida objetiva de maciez) é possível verificar resultados discordantes. Trabalhando com 30 cordeiros inteiros e 30 cordeiros castrados, provenientes do cruzamento de carneiros Hampshire com ovelhas mestiças, destinados a um dos grupos de peso ao abate, 36, 45 e $54 \mathrm{~kg}, \mathrm{KEMP}$ et al. [23] encontraram que a FC diminui com o aumento do peso em ambas as condições sexuais. Num trabalho seguinte, KEMP et al. [21], utilizando os mesmos grupos de peso ao abate, porém, com genótipos diferentes, ou seja, progênies do cruzamento Hampshire $\times$ (Suffolk $\times$ Rambouillet), também encontraram diminuição da FC com o aumento do peso ao abate. Entretanto, outros autores não verificaram efeitos significativos. KEMP, MAHYUDDIN, ELY [22] encontraram que o músculo longissimus dorsi de cordeiros $1 / 2$-Hampshire, $1 / 4$-Suffolk e $1 / 4$ - Rambouillet, não apresentaram diferença nos valores de FC nos grupos de peso ao abate de 32,41 e $50 \mathrm{~kg}$, embora houvesse uma pequena tendência dos valores de $\mathrm{FC}$ diminuírem com o aumento do peso ao abate. PURCHAS, O'BRIEN, PENDLETON [37] relataram que a FC para o músculo biceps femoris não diferiu entre os grupos de peso ao abate de $30,40,50$ e $60 \mathrm{~kg}$, mas para o músculo semimembranosus os valores de FC diminuíram em decorrência do aumento do peso ao abate, mas essa diferença foi irrelevante para alterar a qualidade final da carne. KISHORE, RAWAT, BASUTHAKUR [24] comparando o cruzamento Dorset $x$ Avikalin $(1,86 \mathrm{~kg})$ e somente Avikalin $(1,75 \mathrm{~kg})$ e PEREZ et al. [34], avaliando as raças Santa Inês $(4,51 \mathrm{~kg})$ e Bergamácia $(3,88 \mathrm{~kg})$, não encontraram diferença quanto à maciez. No entanto, SAÑUDO et al. [39], comparando três pesos de carcaças $(8,1 ; 10,2$ e 13,4kg), encontraram que o grupo de peso de carcaça intermediário possuiu maior FC $(4,77 \mathrm{~kg})$ do que o grupo de carcaça mais leve $(3,42 \mathrm{~kg})$ e pesada $(3,44 \mathrm{~kg})$, os quais foram de maciez similar. Os autores sugerem que o grupo de carcaça mais leve possuiu uma boa relação de solubilidade/insolubilidade do colágeno e o mais pesado apresentou uma maior quantidade de gordura intramuscular.

Avaliando a maciez entre espécies, BABIKER, KHIDER, SHAFIE [6] observaram que o músculo 
longissimus dorsi de ovinos $(3,6 \mathrm{~kg})$ e caprinos $(4,0 \mathrm{~kg})$ mostraram-se com valor de FC similar, porém, SCHÖNFELDT et al. [42] encontraram que tanto o músculo longissimus thoracis et lumborum, como o semimembranosus de ovinos, apresentaram menor valor de FC que caprinos das raças Angora e Boer.

O sistema de manejo, na qual são submetidos os ovinos durante a fase de produção, pode afetar a maciez. Segundo AALHUS, PRICE [1], a maciez determinada pelo Warner-Blatzler para os músculos vastus lateralis foi menor para o grupo de ovinos que foram submetidos a exercícios $(4,69 \mathrm{~kg})$ em comparação ao grupo controle não exercitado $(4,97 \mathrm{~kg})$. Os autores supõem que esses resultados são conseqüência da hipertrofia muscular em ovinos exercitados ou um relativo aumento das proteínas miofibrilares, com concomitante diminuição do conteúdo de colágeno por grama de músculo. Resultados semelhantes foram mencionados por NOTTER, KELLY, BERRY [28] que relataram que cordeiros mantidos em diferentes sistemas de manejo apresentaram diferença nos valores de FC, onde a maciez decresceu na ordem, sistema confinado $(5,84 \mathrm{~kg})$, semiconfinado $(5,57 \mathrm{~kg})$ e extensivo $(5,34 \mathrm{~kg})$. No entanto, a repetição desse experimento no ano seguinte não apresentou diferenças na FC.

Cordeiros castrados possuem menor $\mathrm{FC}$ do que cordeiros inteiros [32]. MAIORANO et al. [27], verificaram que cordeiros castrados $(1,64 \mathrm{~kg})$ apresentaram maior maciez, seguido por cordeiros implantados com Zeranol $(2,01 \mathrm{~kg})$ e mostraram menor maciez os cordeiros inteiros $(2,45 \mathrm{~kg})$. No entanto, PURCHAS [36] relatou que diferenças entre ovelhas, machos inteiros e castrados são pequenas ou inconsistentes.

O objetivo do presente trabalho foi avaliar os parâmetros físico-químicos: $\mathrm{pH}$; cor; perda de peso por cozimento; e maciez objetiva (força de cisalhamento), em cordeiros das raças Santa Inês e Bergamácia, distribuídos em quatro grupos de peso ao abate.

\section{2 - MATERIAIS E MÉTODOS}

\section{1 - Análises e medidas realizadas}

\subsection{1 - pH}

A leitura de $\mathrm{pH}$ foi realizada com o auxílio de um pHmetro digital portátil, marca Digimed modelo DM20, dotado de eletrodo de inserção, com resolução de 0,01 unidades de $\mathrm{pH}$. Para a obtenção do valor de $\mathrm{pH}$ foi feita uma pequena incisão no músculo e, em seguida, o eletrodo de vidro foi colocado no músculo até sua estabilização (30 segundos). Foram obtidas três leituras por músculo para cada horário, sendo utilizado na análise estatística o valor médio desses resultados.

Decorrida 1 hora da sangria dos ovinos, a medida de $\mathrm{pH}$ foi realizada nos músculos longissimus dorsi e semimembranosus (esquerdo da carcaça), nos horários de: $1,2,3,4,5,6,8,10,12,15,18$ e 24 horas post mortem.

\subsection{2 - Cor}

A cor do músculo (Sistema colorimétrico CIE L*a*b) foi determinada com a utilização de um colorímetro Minolta Chroma Meter, CR-200b, calibrado para um padrão branco em ladrilho [11].

Os músculos longissimus dorsi em sua região lombar e semimembranosus foram descongelados por 24 horas à temperatura de $6^{\circ} \mathrm{C}$. Foram realizadas leituras em três cortes dentro do mesmo músculo e em três pontos distintos dentro de cada corte. O valor médio desses resultados foi utilizado na análise estatística.

\subsection{3 - Perda de peso por cozimento (PPC)}

Foram utilizadas para análise de PPC, três fatias de cada um dos músculos longissimus dorsi em sua região lombar e semimembranosus, sendo que cada fatia apresentava $2,5 \mathrm{~cm}$ de espessura. As amostras foram pesadas em balanças semi-analítica (Mettler M P1210), embaladas em papel alumínio e assadas em chapa préaquecida a $150^{\circ} \mathrm{C}$. Ao atingir $35^{\circ} \mathrm{C}$, as amostras foram viradas e mantidas assim até a temperatura interna atingir $72 \pm 2^{\circ} \mathrm{C}$ (a temperatura foi monitorada com auxílio de um termômetro digital). Em seguida, as mesmas amostras foram resfriadas à temperatura ambiente e novamente pesadas. A diferença entre peso inicial e peso final determinou a PPC [2].

\subsection{4 - Força de cisalhamento (FC)}

As mesmas amostras utilizadas para a PPC foram usadas para a análise de maciez objetiva. Foram retirados 4 cilindros por fatia de músculo, totalizando 12 cilindros por músculo. Os cilindros foram retirados no sentido da fibra (livre de gorduras e nervos) com o auxílio de uma furadeira acoplada a uma sonda de $1,5 \mathrm{~cm}$ de diâmetro. A FC foi registrada pelo aparelho Instron modelo 1122, acoplado a um acessório Warner-Bratzler, numa escala de 0 a 10 [49].

\section{2 - Análise estatística}

Para análise de $\mathrm{pH}$ utilizou-se um delineamento inteiramente casualizado numa estrutura em parcela subdividida no tempo. O experimento utilizou seis repetições para a raça Santa Inês e três repetições para a raça Bergamácia. $A$ análise de variância foi realizada com auxílio do pacote computacional SAS [41].

Para as demais análises foi usado o delineamento inteiramente casualizado em esquema fatorial com oito tratamentos, quatro grupos de peso ao abate e duas raças. A raça Santa Inês teve seis repetições por tratamento e a raça Bergamácia três repetições, sendo a unidade experimental composta por um animal. O programa estatístico utilizado foi o SAEG [16].

Todas as medidas foram submetidas à análise de regressão, determinadas com o auxílio do programa TableCurve v. 2.03 (Jandel Scientific, incorporatinon) e FCalc 32 for Windows V.11. 

foi:

O modelo estatístico utilizado para a medida de $\mathrm{pH}$

$Y_{i j k l}=\mu+r_{i}+p_{j}+r p_{i j}+e_{(i j) k}+h_{l}+r h_{i l}+p h_{j l}+r p h_{i j l}+e_{i j k l}$

$(i=1,2 ; j=1,2,3,4 ; I=1,2, \ldots 11,12, k=1, \ldots, r)$

Onde:

$\mathrm{Y}_{\mathrm{ijkl}}=$ valor de $\mathrm{pH}$ da raça $\mathrm{i}$ no grupo de peso ao abate $\mathrm{j}$ e horário de medição do $\mathrm{pH}$ I na repetição k;

$\mu=$ média geral;

$r_{i}=$ efeito da raça $i$

$\mathrm{p}_{\mathrm{j}}=$ efeito do grupo de peso ao abate $\mathrm{j}$;

$\mathrm{rp}_{\mathrm{ij}}=$ efeito da interação entre raça i e grupo de peso ao abate j;

$\mathrm{e}_{(\mathrm{ij}) \mathrm{k}}=$ erro (a);

$\mathrm{h}_{\mathrm{l}}=$ efeito do horário de medição do $\mathrm{pH} \mathrm{I}$;

$\mathrm{rh}_{\mathrm{il}}=$ efeito da interação entre raça i e horário de medição do $\mathrm{pH} \mathrm{I}$;

$\mathrm{ph}_{\mathrm{jl}}=$ efeito da interação entre grupo de peso ao abate $\mathrm{j}$ e horário de medição do $\mathrm{pH} \mathrm{I;}$

$\mathrm{rph}_{\mathrm{ijl}}=$ efeito da interação entre raça $\mathrm{i}$, grupo de peso ao abate j e horário de medição do $\mathrm{pH} \mathrm{I}$;

$\mathrm{e}_{\mathrm{ijkl}}=$ erro (b).

O modelo estatístico utilizado para os demais parâmetros físico-químicos foi:

$\mathrm{Y}_{\mathrm{ijk}}=\mu+\mathrm{a}_{\mathrm{i}}+\mathrm{b}_{\mathrm{j}}+(\mathrm{ab})_{\mathrm{ij}}+\mathrm{e}_{(\mathrm{ij}) \mathrm{k}}$

$(i=1,2 ; j=1,2,3,4 ; k=1, \ldots, r)$. Onde:

$Y_{i j k}=$ parâmetro físico-químico da carne na raça i e grupo de peso ao abate j, na repetição k;

$\mu=$ efeito da média;

$a_{i}=$ efeito da raça $i$;

$b_{j}=$ efeito do grupo de peso ao abate j;

$(a b)_{i j}=$ efeito da interação entre raça i e grupo de peso ao abate j;

$\mathrm{e}_{(\mathrm{ij}) \mathrm{k}}=$ erro aleatório.

\section{3 - RESULTADOS E DISCUSSÃO}

\section{1 - Declínio do pH}

A análise estatística para a medida do declínio do $\mathrm{pH}$ no músculo longissimus dorsi encontra-se na Tabela 1.

As raças Santa Inês e Bergamácia apresentaram resultados similares de $\mathrm{pH}$ entre os grupos de pesos estudados. Esses resultados concordam com os trabaIhos de SINNETT-SMITH et al. [45], que estudaram as raças East Friesland, Oxford e Texel; e DRANSFIELD et al. [15] que avaliaram as raças Dorset e Suffolk. Nesses artigos foram encontradas diferenças de $\mathrm{pH}$ entre as raças. No entanto, PEREZ et al. [34] observaram dife- rença nos valores de $\mathrm{pH}$ às 6,12 e 18 horas entre as raças Santa Inês e Bergamácia. Não encontrando efeito de raça apenas aos 30 minutos e 24 horas post mortem, isso talvez possa ser explicado pelo fato de que para a obtenção de tais resultados houve uma associação entre medidas dos músculos longissimus dorsi e biceps femoris e no presente trabalho os músculos longissimus dorsi e semimembranosus foram considerados separadamente.

TABELA 1. Análise de variância para o efeito de raça, grupo de peso ao abate, horário de medição do $\mathrm{pH}$ e respectivas interações, para avaliação do declínio do pH post mortem.

\begin{tabular}{|c|c|c|c|}
\hline \multicolumn{4}{|c|}{ Declínio do $\mathrm{pH}$ no músculo longissimus dorsi $\mathrm{Cv}=2.16$} \\
\hline Fontes de Variação & G.L. & Q.M. & Significância \\
\hline Raça & 1 & 0.02122 & NS \\
\hline Peso & 3 & 1.48645 & 0.0011 \\
\hline Raça*Peso & 3 & 0.09361 & NS \\
\hline ERRO(A) & 28 & 0.20901 & \\
\hline Horas & 11 & $\mathbf{2 . 4 3 8 7 7}$ & 0.0001 \\
\hline Raça*Horas & 11 & 0.02022 & NS \\
\hline Peso*Horas & 33 & 0.04366 & 0.0001 \\
\hline Raça*Peso*Horas & 33 & 0.01721 & NS \\
\hline Erro & 294 & 0.01671 & \\
\hline \multicolumn{4}{|c|}{ Declínio do $\mathrm{pH}$ no músculo semimembranosus $\mathrm{cv}=2.1$} \\
\hline Fontes de Variação & G.L. & Q.M. & Significância \\
\hline Raça & 1 & 0.13290 & NS \\
\hline Peso & 3 & 0.29403 & NS \\
\hline Raça*Peso & 3 & 0.14968 & NS \\
\hline $\operatorname{ERRO}(\mathbf{A})$ & 28 & 0.18151 & \\
\hline Horas & 11 & 2.36068 & 0.0001 \\
\hline Raça*Horas & 11 & 0.07583 & 0.0001 \\
\hline Peso*Horas & 33 & 0.02564 & 0.0144 \\
\hline Raça*Peso*Horas & 33 & 0.01833 & 0.2194 \\
\hline Erro & 294 & 0.01532 & \\
\hline
\end{tabular}

Na Figura 1 está representado o gráfico contendo a equação de regressão entre valor de $\mathrm{pH}$ e horário de medição. O declínio do $\mathrm{pH}$ apresentou comportamento exponencial, indicando uma rápida queda nas primeiras horas post mortem, seguido de estabilização. $O$ coeficiente de determinação (R2) indicou ajustamento eficiente dos dados em torno da curva de regressão.

Analisando o comportamento das curvas de queda de $\mathrm{pH}$ no músculo longissimus dorsi (Figura 1), observa-se que a velocidade do declínio do $\mathrm{pH}$ foi mais rápida nos grupos de cordeiros com peso de 35 e $45 \mathrm{~kg}$, do que nos grupos de 15 e $25 \mathrm{~kg}$. Isso significa que a glicólise desenvolveu-se mais rapidamente em lombos de cordeiros mais pesados. É possível que a velocidade da glicólise tenha variado em função da quantidade de gordura subcutânea entre os grupos de peso ao abate. Os cordeiros dos grupos de 15 e $25 \mathrm{~kg}$ estão em crescimento prioritário de ossos e músculos, já nos grupos de pesos abrangendo os 35 e $45 \mathrm{~kg}$ começa haver desaceleração do crescimento ósseo e muscular com 
concomitante aumento da deposição de gordura. A gordura pode ter agido como isolante térmico [46], fazendo com que a temperatura da carcaça fosse mantida alta por mais tempo nos cordeiros mais pesados. Segundo BOWLING et al. [9] e JOHNSON et al. [19], quanto maior a temperatura da carcaça no post mortem maior a velocidade de glicólise e mais rápida é a queda do $\mathrm{pH}$.

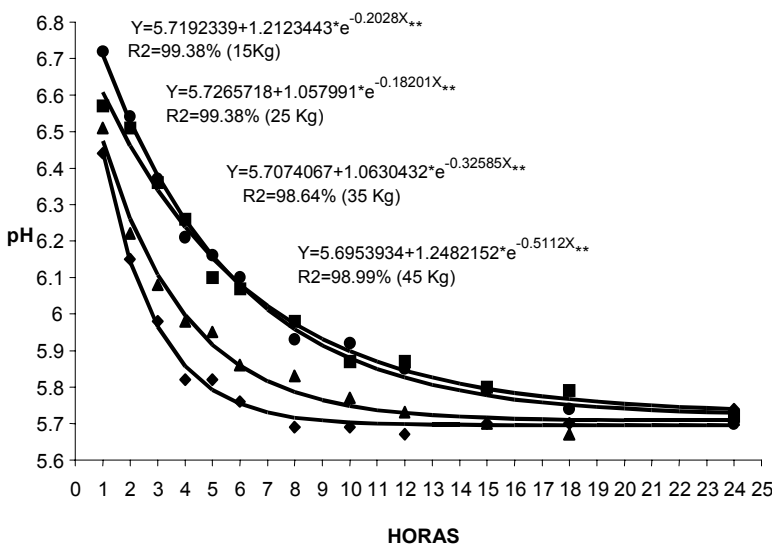

FIGURA 1. Curva de queda do $\mathrm{pH}$ no músculo longissimus dorsi de cordeiros das raças Santa Inês e Bergamácia segundo o grupo de peso ao abate de (l) $15 \mathrm{~kg}$; (n) $25 \mathrm{Kg}$; (s) $35 \mathrm{~kg}$ e (u) $45 \mathrm{~kg}\left({ }^{* *} \mathrm{P}<0,01\right)$.

Esses dados concordam com os resultados de TUMA et al. [48] que observaram a mesma tendência de $\mathrm{pH}$ em relação à idade de abate de novilhos. No entanto, SAÑUDO et al. [39], estudando três grupos de peso de carcaça: 8,1, 10,2 e 13,4kg, encontram que o grupo de carcaça mais pesado apresentou maior valor de $\mathrm{pH}_{\mathrm{f}}$, do que os grupos de carcaça com peso intermediário e leve, os quais foram equivalentes. Os autores atribuíram esses resultados à variação na concentração de glicogênio muscular no momento do abate.

O músculo semimebranosus apresentou interação entre raça e horário de medição do pH (Tabela 1 e Figura 2). E, também, apresentou interação entre grupo de peso ao abate e horário de medição do pH (Tabela 1 e Figura 3). Todas as curvas mostraram comportamento exponencial e os coeficientes de determinação (R2) indicaram um ajustamento eficiente dos dados em torno da curva de regressão.

Na Figura 2, observa-se que a raça Bergamácia apresentou maior velocidade de glicólise até às 8 horas post mortem, momento em que os valores de $\mathrm{pH}$ se igualaram entre as raças, permanecendo dessa maneira, até às 24 horas post mortem. Esses resultados concordam com os achados de PEREZ et al. [34], que descreveram que a raça Bergamácia apresenta maior velocidade de declínio do $\mathrm{pH}$ do que a raça Santa Inês.

Na Figura 3, observa-se que os grupos de peso de 15,25 e $35 \mathrm{~kg}$ apresentaram curvas de declínio do $\mathrm{pH}$ semelhantes, mas o grupo de $45 \mathrm{~kg}$ apresentou maior velocidade de queda do $\mathrm{pH}$. A maior quantidade de gordura nas carcaças no grupo de $45 \mathrm{~kg}$ possivelmente manteve a temperatura das carcaças elevada por mais tempo, promovendo uma aceleração da glicólise, que resultou em uma redução mais rápida do $\mathrm{pH}$.

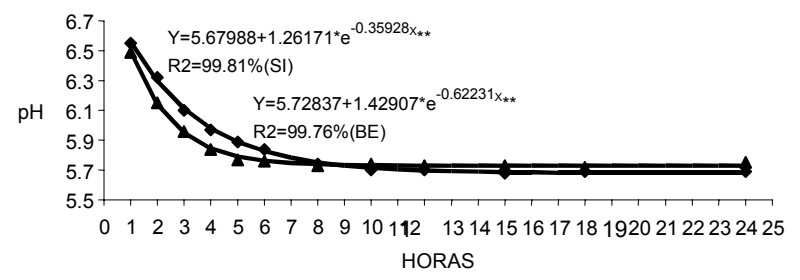

FIGURA 2. Curva de queda do pH no músculo semimembranosus de cordeiros das raças (u) Santa Inês (SI) e (s) Bergamácia (BE) $\left({ }^{* *} \mathrm{P}<0,01\right)$.

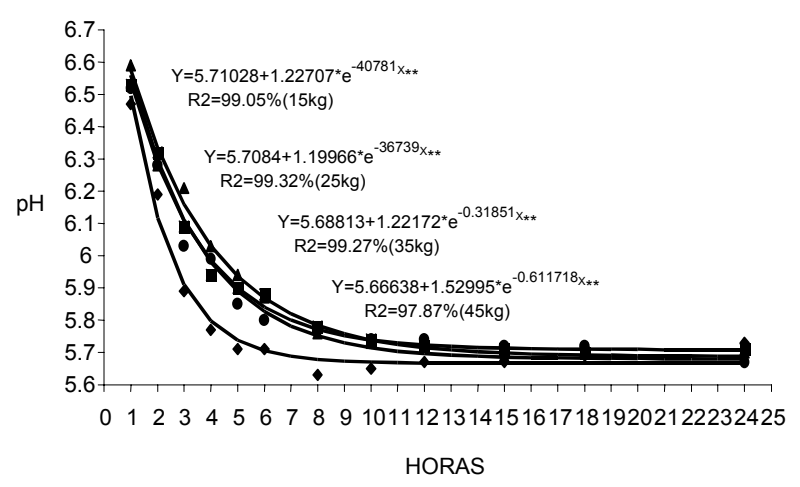

FIGURA 3. Curva de queda do $\mathrm{pH}$ no músculo semimembranosus de cordeiros das raças Santa Inês e Bergamácia segundo o grupo de peso ao abate de (l) $15 \mathrm{~kg}$; (n) $25 \mathrm{Kg}$; (s) $35 \mathrm{~kg} \mathrm{e}(u) 45 \mathrm{~kg}\left({ }^{* *} \mathrm{P}<0,01\right)$.

O músculo longissimus dorsi é relativamente uniforme quanto à profundidade de inserção, diâmetro e é um músculo longo, contribuindo para que possam ser realizadas medidas padronizadas de $\mathrm{pH}$. Por outro lado, o músculo semimembranosus é assimétrico com espessura e profundidade de inserção variável. Isso dificulta a medição do $\mathrm{pH}$, sobretudo nos animais do grupo de peso mais leve, onde a área de exposição do músculo é menor para realização das medidas. Essas particularidades do semimembranosus podem ter contribuído para a diferenciação no comportamento da queda do $\mathrm{pH}$ entre as raças e grupos de peso ao abate.

Os valores de $\mathrm{pH}$ encontrados nos músculos estudados longissimus dorsi e semimembranosus, dessa pesquisa situam-se abaixo ou no limite inferior da faixa de variação dos valores apresentados na literatura, indicando que a glicólise desenvolveu-se mais rapidamente. Tal fato pode ser explicado em função dos animais terem sido abatidos sem insensibilização e, segundo SHORTHOSE [44], cordeiros não insensibilizados possuem maior atividade muscular momentos antes do abate, o que gera maior temperatura muscular, provocando mudanças nos metabólitos do músculo. $E$, também, o abate foi realizado em época quente do ano associado a um intervalo médio de 3 horas entre evisceração e 
início do resfriamento das carcaças. Temperaturas elevadas no post mortem aceleram as reações de glicólise [19].

Embora tenha sido detectada diferença no declínio

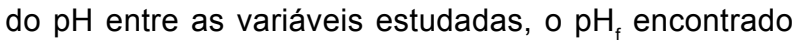
variou de 5,70 a 5,74 no longissimus e de 5,67 a 5,75 no semimembranosus, dentro da faixa de $\mathrm{pH}_{\mathrm{f}}$ considerada normal situada entre 5,5 a 5,8 [18].

\section{2 - Cor}

A análise estatística dos componentes de cor dos músculos longissimus dorsi e semimembranosus é encontrada nos anexos, na Tabela 2.

TABELA 2. Análise de variância para o efeito de raça, grupo de peso ao abate e respectiva interação, para os componentes de cor $\left(\mathrm{L}^{*} \mathrm{a}^{*} \mathrm{~b}^{*}\right)$ nos músculo semimembranosus e longissimus dorsi.

\begin{tabular}{|c|c|c|c|}
\hline \multicolumn{4}{|c|}{ Luminosidade $\left(\mathrm{L}^{*}\right)$ longissimus dorsi $\mathrm{cv}=6.6$} \\
\hline Fontes de Variação & G.L & Q.M & Significância. \\
\hline Raça & 1 & 55.9287 & 0.00476 \\
\hline Peso & 3 & 51.3720 & 0.00033 \\
\hline Raça*Peso & 3 & 5.6259 & NS \\
\hline Resíduo & 28 & 5.9473 & \\
\hline \multicolumn{4}{|c|}{ Vermelho (a*) longissimus dorsi $\mathrm{cv}=10.7$} \\
\hline Fontes de Variação & G.L & Q.M & Significância. \\
\hline Raça & 1 & 3.54664 & NS \\
\hline Peso & 3 & 18.15684 & 0.00007 \\
\hline Raça*Peso & 3 & 0.21280 & NS \\
\hline Resíduo & 28 & 1.69018 & \\
\hline \multicolumn{4}{|c|}{ Amarelo (b*) longissimus dorsi $\mathrm{cv}=13.29$} \\
\hline Fontes de Variação & G.L & Q.M & Significância. \\
\hline$\overline{\text { Raça }}$ & 1 & 0.30175 & NS \\
\hline Peso & 3 & 3.00634 & NS \\
\hline Raça*Peso & 3 & 0.20969 & NS \\
\hline Resíduo & 28 & 1.06282 & \\
\hline \multicolumn{4}{|c|}{ Luminosidade $\left(\mathrm{L}^{*}\right)$ semimembranosus $\mathrm{cv}=7.1$} \\
\hline Fontes de Variação & G.L & Q.M & Significância. \\
\hline$\overline{\text { Raça }}$ & 1 & 37.98782 & 0.02703 \\
\hline Peso & 3 & 74.76797 & 0.00007 \\
\hline Raça*Peso & 3 & 3.43911 & NS \\
\hline Resíduo & 28 & 6.97619 & \\
\hline \multicolumn{4}{|c|}{ Vermelho (a*) semimebranosus $\mathrm{cv}=10.4$} \\
\hline Fontes de Variação & G.L & Q.M & Significância. \\
\hline$\overline{\text { Raça }}$ & 1 & 4.06902 & NS \\
\hline Peso & 3 & 8.99396 & 0.00326 \\
\hline Raça*Peso & 3 & 0.70451 & NS \\
\hline Resíduo & 28 & 1.55139 & \\
\hline \multicolumn{4}{|c|}{ Amarelo (b*) semimembranosus cv=14.1 } \\
\hline Fontes de Variação & G.L & Q.M & Significância. \\
\hline Raça & 1 & 2.17937 & NS \\
\hline Peso & 3 & 3.76966 & 0.02956 \\
\hline Raça*Peso & 3 & 0.40120 & NS \\
\hline Resíduo & 28 & 1.08958 & \\
\hline
\end{tabular}

Os dados para a composição da cor obtidos nos músculos longissimus dorsi e semimembranosus são mostrados nas Figuras 4, 5, 6 e 7. Analisando o coeficiente de determinação (R2) para os resultados de cor, observa-se um eficiente ajustamento dos dados em torno da curva de regressão.

Analisando as Figuras 4 e 5, observa-se que a raça Bergamácia apresentou maior índice de luminosidade nos músculos longissimus dorsi e semimembranosus do que a raça Santa Inês. Ambas as raças tiveram ajustamento de seus dados através de uma equação de regressão linear negativa, indicando que o índice de luminosidade diminuiu com o aumento do peso ao abate.

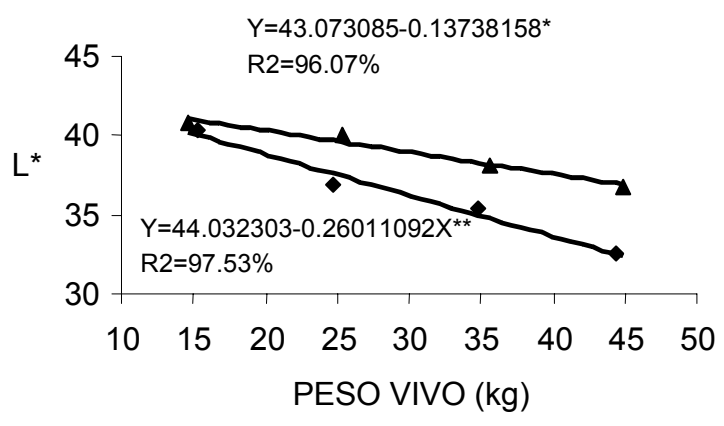

FIGURA 4. Comportamento dos valores de luminosidade $\left(L^{*}\right)$ no músculo longissimus dorsi de cordeiros das raças $(u)$ Santa Inês e (s) Bergamácia segundo o grupo de peso ao abate $\left({ }^{*} P<0,05,{ }^{* *} P<0,01\right)$.

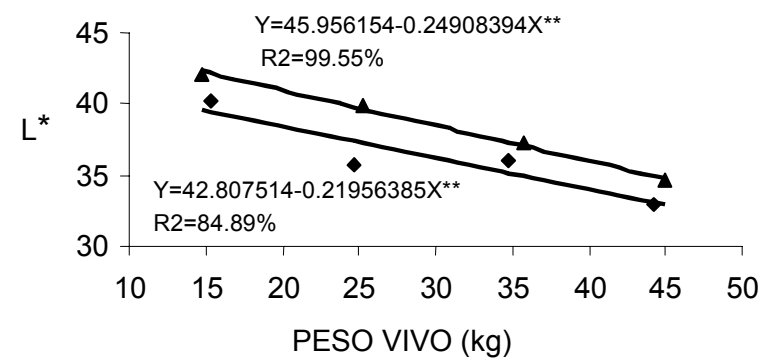

FIGURA 5. Comportamento dos valores de luminosidade ( $\left.L^{*}\right)$ no músculo semimembranosus de cordeiros das raças $(\mathrm{u})$ Santa Inês e (s) Bergamácia segundo o grupo de peso ao abate $\left({ }^{* *} P<0,01\right)$.

A diminuição do valor de $L^{*}$ pode ser explicada pelo conteúdo decrescente do teor de umidade no músculo em função do aumento do peso, fazendo com que ocorra menor luminosidade na superfície dos cortes.

As raças Santa Inês e Bergamácia apresentaram valores similares do teor de vermelho $\left(a^{*}\right)$ nos músculos longissimus dorsi e semimembranosus. Os dados de ambos os músculos ajustaram-se através de uma equação de regressão linear positiva, indicando que com o avanço do peso, aumenta-se a tendência ao vermeIho. Os gráficos contendo esta equação podem ser vistos nas Figuras 6 e 7 para os músculos longissimus dorsi e semimembranosus, respectivamente.

Os valores de composição da cor, encontrados no presente trabalho, variaram: para o valor $L^{*}$ de 32,46 a 42,29; para o valor $a^{*}$ de 10,39 a 13,89; e, para o valor $b^{*}$ de 6,73 a 8,15 . Valores semelhantes foram relatados por FAROUK, PRICE [17], L* $(37,89-37,94)$, a* $(10,64-$ $11,25)$ e $b^{*}(6,34-7,60)$. No entanto, SAÑUDO et al. [39] obtiveram valores de $L^{*}(45,61-48,15)$, a* $(13,4-16,95)$ e $b^{*}(5,90-6,86)$, indicando que as carcaças desse trabalho apresentaram coloração vermelha mais brilhante. Porém, PEREZ et al. [34] encontraram valores de $L^{*}$ $(32,58-32,78)$, a* $(15,58-15,97)$ e $b^{*}(3,38-3,55)$, revelando que as amostras foram mais escuras, do que as amostras avaliadas no presente trabalho. 


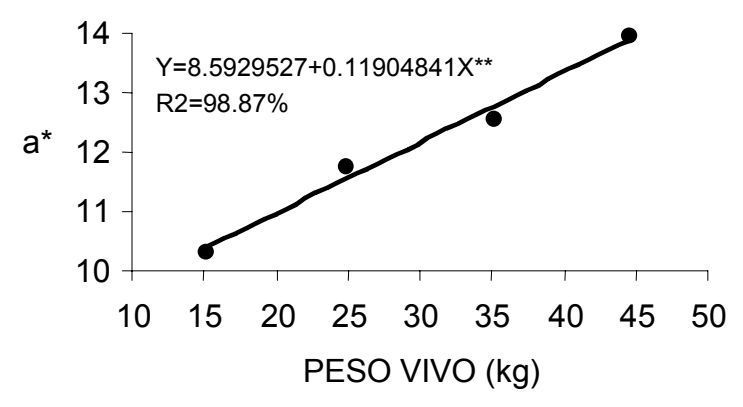

FIGURA 6. Comportamento dos valores de vermelho $\left(a^{*}\right)$ no músculo longissimus dorsi de cordeiros das raças Santa Inês e Bergamácia segundo o grupo de peso ao abate $\left({ }^{* *} P<0,01\right)$.

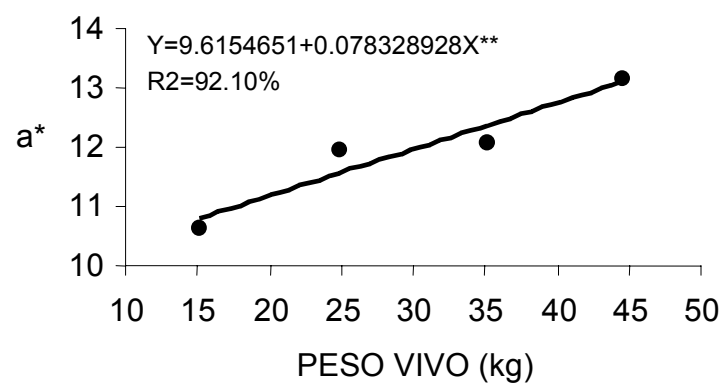

FIGURA 7. Comportamento dos valores de vermelho (a*) no músculo semimembranosus de cordeiros das raças Santa Inês e Bergamácia segundo o grupo de peso ao abate $\left({ }^{\star *} P<0,01\right)$.

Considerando os três componentes de cor, obtidos no trabalho atual, observa-se que $L^{*}$ diminuiu de 42,29

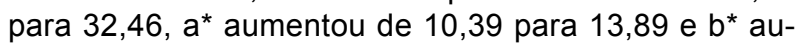
mentou de 6,73 para 8,15 , com o aumento do peso ao abate de $15 \mathrm{~kg}$ para $45 \mathrm{~kg}$. Isso indicou que a carne dos cordeiros apresentam coloração vermelha mais escura com o aumento do peso de abate. Isso pode ser explicado pelo fato dos animais mais pesados possuírem maior massa muscular e, conseqüentemente, maior irrigação sangüínea, maior concentração de proteínas sarcoplasmáticas e outros pigmentos.

Embora a coloração dos músculos longissimus dorsi e semimembranosus do grupo de peso ao abate na faixa dos $45 \mathrm{~kg}$ tenha apresentado coloração vermelha mais escura, o mesmo não foi classificado como corte demasiadamente escuro (DFD, com $\mathrm{pH}_{\mathrm{f}}$ maior do que 6,0), causada por condições de manejo estressantes no préabate. Nesse caso, a cor vermelho escura foi inerente à raça e à idade.

\section{3 - Perda de peso por cozimento (PPC)}

A análise de variância para o efeito de raça, grupo de peso ao abate e respectiva interação para PPC, medida nos músculos longissimus dorsi e semimembranosus, são apresentadas nas Tabelas 3 e 4.

O efeito das raças, assim como os grupos de peso ao abate não apresentaram efeitos significativos sobre a PPC (Tabelas 3 e 4). As médias e desvio-padrão para a PPC nos diferentes grupos de peso e músculos estudados são apresentados na Tabela 5.
TABELA 3. Análise de variância para o efeito de raça, grupo de peso ao abate e respectiva interação, para a perda de peso por cozimento (\%) e força de cisalhamento $(\mathrm{kg})$ no músculo longissimus dorsi.

\begin{tabular}{|c|c|c|c|}
\hline \multicolumn{4}{|c|}{ Perda de peso por cozimento (longissimus dorsi) $\mathrm{cv}=8.5$} \\
\hline $\begin{array}{l}\text { Fontes de } \\
\text { Variação }\end{array}$ & G.L & Q.M. & Significância \\
\hline Raça & 1 & 2.40534 & NS \\
\hline Peso & 3 & 7.92808 & NS \\
\hline Raça*Peso & 3 & 11.45559 & NS \\
\hline Residuo & 28 & 5.63012 & \\
\hline
\end{tabular}

\begin{tabular}{lccc}
\hline \multicolumn{3}{c}{ Força de cisalhamento $($ longissimus dorsi) } & $\mathbf{c v}=\mathbf{2 2}$ \\
\hline $\begin{array}{l}\text { Fontes de } \\
\text { Variação }\end{array}$ & G.L. & Q.M. & Significância \\
\hline Raça & 1 & 0.05210 & \\
Peso & 3 & 0.37548 & NS \\
Raça*Peso & 3 & 0.14439 & NS \\
Residuo & 28 & 0.32512 & \\
\hline
\end{tabular}

TABELA 4. Análise de variância para o efeito de raça, grupo de peso ao abate e respectiva interação, para a perda de peso por cozimento $(\%)$ e força de cisalhamento $(\mathrm{kg})$ no músculo semimembranosus.

\begin{tabular}{lccc}
\hline \multicolumn{4}{c}{ Perda de peso por cozimento (semimembranosus) } \\
\hline Fontes de Variação & G.L. & Q.M. & Significância \\
\hline Raça & 1 & 47.63264 & NS \\
Peso & 3 & 21.41445 & NS \\
Raça*Peso & 3 & 5.60640 & NS \\
Residuo & 28 & 16.08966 & \\
\hline \multicolumn{4}{c}{ Força de cisalhamento (semimembranosus) } \\
\hline Fontes de Variação & G.L. & Q.M. & Significância \\
\hline Raça & 1 & 0.57034 & NS \\
Peso & 3 & 0.93860 & NS \\
Raça*Peso & 3 & 0.10691 & NS \\
Residuo & 28 & 0.39110 & \\
\hline
\end{tabular}

TABELA 5. Resultados da percentagem (PPC) e respectivo desvio padrão, encontrado no músculo longissimus dorsi (LD) e semimembranosus (SM), segundo os grupos de peso ao abate.

\begin{tabular}{lcccc}
\hline \multicolumn{5}{c}{ Grupos de peso ao abate } \\
\cline { 2 - 5 } & $15 \mathrm{~kg}$ & $25 \mathrm{~kg}$ & $35 \mathrm{~kg}$ & $45 \mathrm{~kg}$ \\
\hline LD & $27,6 \pm 3,2$ & $27,2 \pm 2,3$ & $29,1 \pm 2,2$ & $28,1 \pm 1,9$ \\
SM & $31,1 \pm 4,4$ & $30,3 \pm 2,9$ & $33,1 \pm 4,5$ & $29,4 \pm 4,1$ \\
\hline
\end{tabular}

A similaridade das medidas de PPC obtidas no presente trabalho entre as raças Santa Inês e Bergamácia está de acordo com os dados descritos por PEREZ et al. [34], nos músculos longissimus e biceps femoris.

Quanto à idade e/ou peso ao abate, LLOYD, SLYTER, COSTELLO [26] estudando ovinos com 54 e $64 \mathrm{~kg}$, e KADIM et al. [20] avaliando linhagens ovinas selecionadas para alta e baixa quantidade de gordura, também não encontraram diferença na PPC. No entanto, KEMP et al. [21, 23] e SCHÖNFELDT et al. [42] relataram diferenças na PPC, que aumentou conforme o aumento do peso dos cordeiros. Essas diferenças encontradas, sob condições similares de cozimento, foram atribuídas à quantidade de gordura existente na carne.

No presente trabalho, não foi encontrada diferença entre os grupos de peso ao abate em relação à $\mathrm{PPC}$, isso pode ser explicado sob dois pontos: primeiro tanto o músculo longissimus dorsi como o semimembrano- 
sus tiveram a capa de gordura externa removida; segundo, como se trata de animais em crescimento (mesmo os pertencentes ao grupo mais pesado), não houve tempo suficiente para ocorrer uma deposição de gordura intramuscular (marmoreio). Os resultados da PPC encontrada nessa pesquisa variaram de 27,2 a $33,1 \%$. Valores maiores para PPC em carne ovina foram relatados por OCKERMAN et al. [29], BABIKER, EL KHIDER, SHAFIE [6] e PEREZ et al. [34] com variação entre 35,03 a $41,05 \%$. Resultados médios menores foram descritos por KEMP et al. [21,23] e APPLE et al. [4], cuja variação foi de 14,1 a $24,5 \%$. Entretanto, resultados similares foram encontrados por ASGHAR, YEATES [5] e KADIM et al. [20], com variação de 25, 99 a 31,6\%.

Essas variações para valores de PPC entre os vários autores podem ser atribuídas principalmente a diferenças no genótipo, condições de manejo pré e pósabate dos ovinos, metodologia no preparo das amostras, tais como a remoção ou padronização da capa de gordura externa e tipo de equipamento onde podem variar a temperatura no processo de cocção.

\section{4 - Força de cisalhamento (FC)}

A análise de variância para o efeito de raça, grupo de peso ao abate e respectiva interação para $\mathrm{FC}$, medida nos músculos longissimus dorsi e semimembranosus, são apresentadas nas Tabelas 3 e 4.

As raças Santa Inês e Bergamácia, assim como os grupos de peso ao abate apresentaram valores similares de FC em ambos os músculos estudados (Tabela 6).

TABELA 6. Resultado da força de cisalhamento (em $\mathrm{kg}$ ) e respectivo desvio padrão, encontrado no músculo longissimus dorsi (LD) e semimembranosus (SM), segundo os grupos de peso ao abate.

\begin{tabular}{lcccc}
\hline \multicolumn{5}{c}{ Grupos de peso ao abate } \\
\cline { 2 - 5 } & $15 \mathrm{~kg}$ & $25 \mathrm{~kg}$ & $35 \mathrm{~kg}$ & $45 \mathrm{~kg}$ \\
\hline LD & $2,7 \pm 0,5$ & $2,3 \pm 0,5$ & $2,8 \pm 0,7$ & $2,5 \pm 0,6$ \\
SM & $2,6 \pm 0,6$ & $2,5 \pm 0,5$ & $3,1 \pm 0,5$ & $3,2 \pm 0,7$ \\
\hline
\end{tabular}

A variação dos valores de $\mathrm{FC}$ encontrada nesse trabalho foi de 2,3 a 3,2kg. Valores maiores foram relatados por BABIKER, EL KHIDER, SHAFIE [6], AALHUS, PRICE [1] e APPLE et al. [3], cuja variação foi de 3,60 a $5,30 \mathrm{~kg}$. No entanto, resultados aproximados foram descritos por CLARE et al. [12] com variação de 2,81 a $3,00 \mathrm{~kg}$. Essas divergências nos valores de FC ocorrem por inúmeros motivos, como por exemplo: manejo empregado no pré-abate, velocidade na instalação do rigor mortis, pH no post mortem, temperatura pré-abate, instalação e extensão da glicólise, músculo utilizado, manejo pós-abate (como estimulação elétrica e desossa a quente), condições de acondicionamento e metodologia para as determinações, tais como: temperatura e tempo empregado no processo de cocção.

KNAPP et al. [25] atribuíram limites para a FC de carne bovina, onde valores acima de $4,5 \mathrm{~kg}$ foram considerados amostras duras e inaceitáveis, enquanto FC abaixo de 4,5kg foi descrita como macia e aceitável pelo consumidor. Assumindo essa classificação, podese considerar que os resultados de medida de FC para os músculos longissimus dorsi e semimembranosus encontrados nesse trabalho são classificados como macio, portanto, de alta aceitabilidade.

A comparação da maciez entre as raças ovinas (Santa Inês e Bergamácia) é justificada pelas diferenças para esse atributo entre os genótipos. Em bovinos, diversos estudos revelam que a participação de genótipos das raças zebuínas (Bos indicus), tende a afetar negativamente a maciez da carne [30].

Comparando os resultados obtidos nesse trabalho com aqueles descritos na literatura é possível verificar que a similaridade nos valores de FC entre as raças Santa Inês e Bergamácia foi relatada por PEREZ et al. [34], porém com valores maiores $(4,51$ e $3,88 \mathrm{~kg})$ do que os encontrados neste trabalho. Outros autores também não citaram diferenças para FC, tais como: NOTTER, KELLY, BERRY [28] comparando as progênies do cruzamento de ovelhas $1 / 2$-Suffolk, $1 / 2$-Rambouillet; $1 / 2$-Suffolk, $1 / 4$-Finnsheep, 1/4-Rambouillet ou Dorset com machos Suffolk; e KISHORE, RAWAT, BASUTHAKUR [24] que encontraram em cordeiros machos Dorset $x$ Avikalin e Avikalin médias de FC de 1,75 e1, 86kg, respectivamente.

Embora nesta pesquisa os grupos de peso ao abate não tenham apresentado diferença quanto à $\mathrm{FC}, \mathrm{KEMP}$ et al. [21, 23] afirmam que ocorre um aumento da maciez da carne de cordeiros com o aumento do peso ao abate. Os dados de PURCHAS, O'BRIEN, PENDLETON [37] discordam, mostrando que a maciez da carne de cordeiros diminui com o aumento do peso ao abate, porém relataram que essa diferença foi irrelevante para alterar a qualidade final da carne.

A similaridade dos resultados de FC entre os grupos de peso ao abate neste trabalho deve-se ao fato de que os cordeiros utilizados nesse experimento são animais jovens, com idade máxima de 8 meses, e segundo YOUNG, BRAGGINS [50] apesar da solubilidade do colágeno declinar com a idade, sua concentração permanece inalterada dos 4 meses aos 5 cinco anos de idade. Também, DEVINE, CHRYSTALL, DAVEY [14] descreveram que a maciez entre cordeiros jovens a um $\mathrm{pH}_{\mathrm{f}}$ baixo foi similar a cordeiros mais velhos a $u m \mathrm{pH}_{\mathrm{f}}$ alto, concluindo que a maciez do longissimus de ovinos não foi influenciada pelo conteúdo de colágeno.

\section{4 - CONCLUSÕES}

A glicólise post mortem se desenvolveu mais rapidamente com o aumento do peso dos cordeiros.

Os músculos longissimus dorsi e semimembranosus da raça Bergamácia apresentam maior brilho do que os músculos da raça Santa Inês. Esses músculos, de ambas as raças, são mais escuros com o aumento do peso dos ovinos ao abate.

As raças Santa Inês e Bergamácia e os pesos ao abate estudados possuem valores similares para os 
parâmetros perdas de peso por cozimento e força de cisalhamento nas amostras dos músculos longissimus dorsi e semimembranosus.

\section{5 - REFERÊNCIAS BIBLIOGRÁFICAS}

[1] AALHUS, J.L; PRICE, M.A. The effect of a progressive-resistance exercise on growth, development and meat quality of sheep. Can. J. Anim. Sci; Alberta, v. 70, p. 89-95, mar; 1990.

[2] AMASA. Guidelines for Cookingand Sensory Evaluation of Meat. American Meat Science Association, National Live Stock and Meat Board, Chicago, IL. 1978.

[3] APPLE, J.K; DIKEMAN, M.E; MINTON, J.E; McMURPHY, R.M; FEDDE, M.R; LEIGHT, D.E;; Unruh, J.A. Effects of restrain and isolation stress and epidural blockade on endocrine and blood metabolite status, muscle glycogen metabolism, and indice of darck-cutting longissimus muscle of Sheep. Journal of Animal Science, v. 73, p. 2295-2307, 1995.

[4] APPLE, J.K; UNRUH, J.A; MINTON, J.E; BARTLETT, J.L. Influence of repeated restrain and isolation stress and electrolyte administration on caracass quality and muscle electrolyte content of sheep. Meat Science, v. 35, p. 191203, 1993.

[5] ASGHAR, A; YEATES, N.T.M. Muscle characteristicis and meat quality of lambs, grown on different nutritional planes. IV. Effect on meat quality. Agric. Biol. Chem, v. 43, n.3, p. 455-456, 1979.

[6] BABIKER, S.A; EL KHIDER, I.A; SHAFIE, S.A. Chemical composition and quality attributes of goat meat and lamb. Meat Science, v. 28, p. 273-277, 1990.

[7] BARBUT, S. Estimates and detection of the PSE problem in young turkey breast meat. Canadian Journal of Animal Science. Ottawa, v. 76, n. 3, p. 455-4557, 1996.

[8] BOUTON, P.E; HARRIS, P.V; SHORTHOSE, W.R. Effect of ultimate $\mathrm{pH}$ upon the water-holding capacity and tenderness of mutton. Journal of Food Science, v. 36, p. 435-439, 1971.

[9] BOWLING, R.A; SMITH, G.C; DUTSON, T.R; CARPENTER, Z.L. Effects of prerigor conditioning treatments on lamb muscle shortening, $\mathrm{pH}$ and ATP. Journal of Food Science, v. 43, p. 502-507, 1978.

[10] BRESSAN, M.C. Efeito do tempo entre a sangria e a entrada das carcaças na câmara fria e de diferentes velocidades de resfriamento sobre a qualidade da carne suína. Porto Alegre, 1992. 94p. (Dissertação-Mestrado em Zootecnia), Universidade Federal do Rio Grande do Sul (UFRGS).

[11] BRESSAN, M.C. Efeito dos fatores pré e pós-abate sobre a qualidade da carne de peito de frango. Campinas: FEA [s.n.], 1998, 201p. (Tese-Doutorado em Tecnologia de Alimentos), Universidade Estadual de Campinas (UNICAMP).

[12] CLARE, T.L; JACKSON, S.P; MILLER, M.F; ELLIOTT, C.T; RAMSEY, C.B. Improving tenderness of normal and callipyge lambs with calcium chloride. Journal of Animal Science, v. 75, p. 377-385, 1997.

[13] CULAU, P.O.V. Efeito da distância criação-abatedouro e temperatura de descanso pré-abate sobre a qualidade da carne suína. Porto Alegre, 1991, 132p. (Dissertação-Mestrado em Zootecnia), Universidade Federal do Rio Grande do Sul (UFRGS).

[14] DEVINE, C.E; CHRYSTALL, B.B; DAVEY, C.L. Effects of nutrition in lambs and subsequent postmortem biochemical changes in muscle. New Zealand of Agricultural Research, v. 26, p. 53-57, 1983.
[15] DRANSFIELD, E; NUTE, G.R; HOGG, B.W; WALTERS, B.R. Carcass and eating quality of ram, castrated ram and ewe lambs. Anim. Prod; Hamilton, v. 50, p. 291-299, 1990.

[16] EUCLYDES, R.F. Manual de Utilização do Programa SAEG (Sistema para Análise Estatística e Genética) Viçosa: Universidade Federal de Viçosa. 59p. 1983.

[17] FAROUK, M.M; PRICE, J.F. The effect of post-exsanguination infusion on the composition, exudation, color and postmortem metabolic changes in lamb. Meat Science, v. 38, p. 477-496, 1994.

[18] FORREST, J.C; ABERLE, E.D; HEDRICK, HB; JUDGE, M.D; MERKEL, R.A. Fundamentos de ciencia de la carne. Traduzido por BERNABÉ SANZ PÉREZ. Zaragoza. ACRIBIA, S.A. (ed) 1979. 364p. Tradução de: Principles of meat Science.

[19] JOHNSON, M.H; BIDNER, T.D., MCMILLIN, K.W; DUGAS, S.M; HEMBRY, F.G. The effect of three temperature conditioning treatments and subcutaneous fat removal on lamb quality. Journal of Animal Science, v. 67, p. 2309-2315, 1989.

[20] KADIM, I.T; PURCHAS, R.W; DAVIES, A.S; RAE, A.L; BARTON, R.A. Meat quality and muscle fibre type characteristics of Southdown rams from high and low backfat selection lines. Meat Science, v. 33, p. 97-109, 1993.

[21] KEMP, J.D; JOHNSON, A.E; STEWART, D.F; ELY, D.G; FOX, J.D. Effect of dietary protein, slaughter weight and sex on carcass composition, organoleptic properties and cooking losses of lamb. Journal of Animal Science, v. 42, n. 3 , p. 575-583, 1976.

[22] KEMP, J.D; MAHYUDDIN, M; ELY, D.G. Effect of feeding systems, slaughter weight and sex on organoleptic properties, and fatty acid composition of lamb. Journal of Animal Science, v. 51, n. 2, p. 321-330, 1981.

[23] KEMP, J.D; SHELLEY, J.M; ELY, D.G; MOODY, W.G. Effects of castration and slaughter weight on fatness, cooking losses and palability of lamb. Journal of Animal Science, v. 34, n. 4, p. 560-562, 1972.

[24] KISHORE, K; RAWAT, P.S; BASUTHAKUR, A.K. Comparative performace of terminal cross of Avikalin with Dorset and Avikalin lambs regarding growth, feedlot and carcass characteristics. Indian J. Anim. Sci., v. 58, n. 8, p. 774778, august, 1984.

[25] KNAPP, R.H; TERRY, C.A; SAVELL, J.W; CROSS, H.R; MIES, W.L; EDWARDS, J.W. Charcacterization of cattle types to meet specific beef targets. Journal of Animal Science, v. 67, p. 2294-2308, 1989.

[26] LLOYD, W.R; SLYTER, A.L; COSTELLO, W.J. Effect of breed, Sex and final weight on feedlot performace, carcass characteriistics and meat palatability of lambs. Journal of Animal Science, v. 51, n. 2, p. 316-320, 1981.

[27] MAIORANO, G; McCORMICK, R.J; FIELD, R.A; SNOWDER, G. Intramuscular collagen characteristics of ram, wether, and zeranol-implanted ram lambs. Journal of Animal Science, v. 71, 1817-1822, 1993.

[28] NOTTER, D.R; KELLY, R.F; BERRY, B.W. Effects of ewe breed and management system on efficiecy of lamb production: III. Meat Characteistics. Journal of Animal Science, v. 69, p. 3523-3532, 1991.

[29] OCKERMAN, H.W; EMSEN, H; PARKER, C.F; PIERSON, C.J. Influence of type (wooled or hair) and breed on growth and carcass characteristics and sensory properties of lamb. Journal of Food Science, v. 47, p. 1365-1368, 1982.

[30] OLIVEIRA, A.L. Efeito do peso de abate nos rendimentos, características de carcaça e qualidade da carne de noviIhos nelore e mestiços canchim-nelore. Campinas, 1993, 
130p. (Dissertação-Mestrado em Tecnologia de Alimentos), Faculdade de Engenharia de Alimentos, Universidade Estadual de Campinas (UNICAMP).

[31] OSÓRIO, M.T; SIERRA, I; SAÑUDO, C; OSÓRIO, J.C. Influência da raça, sexo e peso/idade sobre o rendimento da carcaça em cordeiros. In: REUNIÃO DA SOCIEDADE BRASILEIRA DE ZOOTECNIA, 35; 1998, Botucatu. Anais... Botucatu: Gnosis, 1998.1 CD-ROM.

[32] PARDI, M.C; SANTOS, I.F. SOUZA, E.R; PARDI, H.S. Ciência, higiene e tecnologia da carne: tecnologia da sua obtenção e transformação. Goiânia: Centro Editorial e Gráfico Universidade de Goiás, v. 1, 1993. 586p.

[33] PEARSON, A.M. La función muscular y los cambios postmortem. In: PRICE, J.F; SCHWEIGERT, B.S. Ciencia de la carne y de los productos carnicos. Cap.4, p. 139174. Tradução de FUENTE, J.L. Zaragoza: ACRIBA S.A. 1994. ed $2^{\circ}$, Tradução de: The science of meat and meat products ed. $3^{\circ}$.

[34] PEREZ, J.R.O; BONAGURIO, S; BRESSAN, M.C; PRADO, O.V. Efeito dos Dejetos de Suíno na Qualidade da Carne de Ovino. In: REUNIÃO DA SOCIEDADE BRASILEIRA DE ZOOTECNIA, 34, Juiz de Fora, 1997. Anais... Juiz de Fora: SBZ, 1997, v. 1, p. 391.

[35] PINKAS, A; MARINOVA, P; TOMOV, I; MONIN, G. Influence of age at slaughter, rearing technique and pre-slaughter treatament on some quality traits of lamb meat. Meat Science, Great Britain, v. 6, p. 245-255, 1982.

[36] PURCHAS, R.W. Effect of Sex and castration on growth and composition. In: PEARSON, A.M; DUTSON, T.R. Growth regulation in farm animals advances in meat research. London: Elsevier, 1996. p. 203-249.

[37] PURCHAS, R.W; O'BRIEN, L.E; PENDLETON, C.M. Some effects nutrition and castration on meat production from male Suffolk cross (Border Leicester-Romney cross) lambs. N. Z. Journal of Agricultural Research, v. 22, p. 375383, 1979.

[38] SAINZ, R.D. Qualidade das Carcaças e da Carne Bovina. In: Congresso Brasileiro das Raças Zebuínas. 27 a 30 de Outubro de 1996. Reprodução e Genética Aplicada aos Zebuínos. 2, 1996, Anais..., 1996, p. 1.

[39] SAÑUDO, C.; SANTOLARIA, M.P.; MARÍA G.; OSORIO, M; SIERRA, I. Influence of caracass weight on instrumental and sensory lamb meat quality in intensive production systems. Meat Science, v. 42, n. 2, p. 195-202, 1996.

[40] SARANTOPOULOS, C.I.G.L. e PIZZINATTO, A. Fatores que afetam a cor das carnes. Coletânea ITAL, Campinas, v. 20, n. 1, p. 1-12, 1990.

[41] SAS-Institute. SAS User's guide: statistics. $5^{\text {a }}$ ed; SAS Inst. Inc; Cary, North Carolina, 1985. 956p.

[42] SCHÖNFELDT, H.C; NAUDÉ, R.T; BOK, W; VAN HEERDEN, S.M; SMIT, R; BOSHOFF, E. Flavour and tenderness-related quality characteristics of goat and sheep meat. Meat Science, v. 34, p. 363-379, 1993.

[43] SHACKELFORD, S.D; PURSER, D.E; SMITH, G.C; GRIFFIN, C.L; STIFFLER, D.M; SAVELL, J.W. Lean color characteristics of bullock and steer beef.. Journal of Animal Science, v. 70, p. 465-469, 1992.

[44] SHORTHOSE, W.R. Effects of level of feeding, pre-slaughter stress and method of slaugter on postmortem glycolysis of sheep muscles. Meat Science, v. 2, n. 3, p. 189-198, 1978.

[45] SINNETT-SMITH, P.A; WOOLLIAMS, J.A; WARRISS, P.D; ENSER, M. Effects of recombinant DNA-derived bovine somatotropin on growth, carcass characteristics and meat quality in lambs from three breeds. Anim. Prod. v. 49, p. 281-289, 1989.

[46] SMITH, G.C; DUTSON, T.R; HOSTETLER, R.L; CARPENTER, Z.L. Fatness, rate of chilling and tenderness of lamb. Journal of Food Science, v. 41, p. 748-756, 1976.

[47] SOLOMON, M.B; KEMP, J.D; MOODY, W.G; ELY, D.G; FOX, J.D. Effect of breed and slaughter weight on physical, chemical and organoleptic properties of lamb carcasses. Journal of Animal Science, v. 51, n. 5, p. 1102-1107, 1980.

[48] TUMA, H.J; HENRICKSON, R.L; ODELL, G.V; STEPHENS, D.F. Varation in the physical and chemical characteristics of the longissimus dorsi muscle from animals differing in age. Journal of Animal Science, v. 22, p. 354-357, 1963.

[49] WHEELER, T.L; KOOHMARAIE, M. Prerigor and postrigor changes in tenderness of ovine longissimus Muscle. Journal of Animal Science, v. 72, p. 1232-1238, 1994.

[50] YOUNG, O.A; BRAGGINS, T.J. Tenderness of ovine semimembranosus: Is collagen concentration or solubility the critical fator? Meat Science, v. 35, p. 213-222, 1993. 